\title{
SURFACE-WAVE INTERACTION WITH A DEEPLY SUBMERGED CIRCULAR DUCT
}

\author{
JOHN W. MILES'
}

(Received 27 May 1982; revised 13 August 1982)

\begin{abstract}
The interaction of a surface wave of angular frequency $\omega$ with a deeply submerged, vertical, open-mouthed, circular duct of radius $a$ is considered. The resulting boundaryvalue problem is solved by the Wiener-Hopf technique. The pressure-amplification factor (the ratio of the complex amplitude of the pressure in the depths of the duct to that of the incident wave in the plane of the mouth) is determined in closed form as a function of the dimensionless wave number $K=\omega^{2} a / g$.
\end{abstract}

\section{Introduction}

Submerged ducts provide a promising mechanism for extracting energy from ocean swell and have been studied extensively in the United Kingdom during the past few years. A description of the basic program has been given by Lighthill [3], and I note here only that the ducts may be designed to resonate with the incoming swell, thereby providing an amplification of the response that at least partially compensates for the diminution associated with the submergence of the mouths (which is required for the integrity of the ducts during storms). The basic theoretical problem is to determine the wave-induced pressure fluctuations that are excited in the depths of a periodic row of submerged, vertical, open-mouthed, circular ducts; however, the analysis for even this idealized model is rather complicated, and it therefore is worthwhile to consider even simpler models.

Lighthill [3] has considered what is perhaps the simplest, non-trivial model, namely a single, deeply submerged, two-dimensional duct (as a limiting case of a single, two-dimensional duct with mouth at finite depth). I consider here a deeply

\footnotetext{
'University of California, La Jolla, California 92093, U.S.A.

(C) Copyright Australian Mathematical Society 1983
} 
submerged circular duct in an ocean of infinite depth (Figure 1). Deeply submerged implies that the effect of the free surface on the interaction between swell and duct is negligible, which assumption presumably is valid for $\omega^{2} h / g \gg 1$, where $\omega$ is the angular frequency of the surface wave and $h$ is the depth of the mouth; the circular duct with unrestricted $\omega^{2} h / g$ has been considered by Simon [6]. The primary reason for the present ("local" in Lighthill's terminology) approximation is the relative simplicity of both the analysis and the results vis-à-vis the original problem; in addition, this approximation provides an excellent trial function for variational approximations for the more complicated problem of a periodic row of ducts (Miles [4]).

I posit the wave-induced pressure in the form

$$
p=\operatorname{Re}\left\{p_{0} \phi(r, z, \theta) e^{i \omega t}\right\},
$$

where $p_{0}$ is a reference pressure, $a$ is the radius of the duct, $\phi$ is the complex amplitude of the dimensionless pressure (hereinafter designated simply as the pressure), and $r, z, \theta$ are dimensionless, cylindrical coordinates with the reference length $a$ and origin in the center of the mouth of the duct (Figure 1). The boundary-value problem for an incompressible, inviscid fluid is prescribed by

$$
\begin{gathered}
\nabla^{2} \phi=0, \\
\phi \sim \exp (-i K r \cos \theta-K z) \equiv \phi_{\infty} \quad(r \rightarrow \infty), \\
\partial_{r} \phi=0 \quad(r=1,0<z<\infty),
\end{gathered}
$$

where $K=\omega^{2} a / g$ is the dimensionless wave number of the incident wave. (Note that $\phi$ may be interpreted as a dimensionless complex velocity potential if $p$ and $p_{0}$ are correspondingly reinterpreted.)

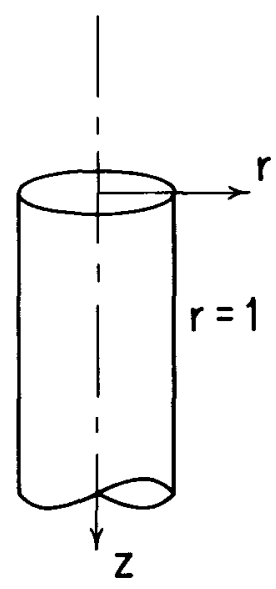

Figure 1. Semi-infinite, circular duct. 
The perturbation pressure induced by the duct, $\phi-\phi_{\infty}$, may be expressed as a functional of the pressure jump

$$
\chi(z, \theta)=\phi(1-, z, \theta)-\phi(1+, z, \theta) .
$$

Continuity and the requirement that the velocity not be more singular than $z^{-1 / 2}$ near the lip of the duct (the latter condition may be inferred from the requirement that the energy in the neighbourhood of the lip be bounded) imply

$$
\chi=0 \quad(-\infty<z<0) ; \quad \chi=O\left(z^{1 / 2}\right) \quad(z \rightarrow 0+) . \quad(1.6 \mathrm{a}, \mathrm{b})
$$

It proves expedient for the subsequent development of the Wiener-Hopf solution to suppose that the fluid is slightly compressible and replace (1.2) by the Helmholtz equation

$$
\left(\nabla^{2}+\varepsilon^{2}\right) \phi=0
$$

where

$$
\varepsilon \equiv \omega a / c=\alpha-i \beta \quad(0<\beta \ll \alpha \ll 1),
$$

$c$ is the sonic speed in the fluid, $\alpha$ is the (dimensionless) acoustical wave number, and $\beta>0$ implies a slow growth of the disturbance from a state of rest at $t=-\infty$ (see Lighthill [2, Section 3.9] for a more detailed description of this artifice). The final results for the incompressible fluid follow through the limit $\alpha, \beta \downarrow 0$.

The pressure-amplification factor, defined as the ratio of the complex amplitude of the pressure in the depths of the duct $(z \rightarrow \infty)$ to that of the incident wave in the plane of the mouth, is given by (I anticipate that $\phi$ tends to a constant as $z \rightarrow \infty$ with $r<1$ and vanishes as $z \rightarrow \infty$ with $r>1$ )

$$
P=\lim _{z \rightarrow \infty} \phi(r, z, \theta)=\chi_{0}(\infty) \quad(r<1),
$$

where $\chi_{0}(z)$ is the axisymmetric component in the Fourier expansion of $\chi(z, \theta)$.

\section{Integral-equation formulation}

The pressure of the incident wave, as given by (1.3), admits the Jacobi-Anger expansion

$$
\phi_{\infty}=e^{-K z} \sum_{n=0}^{\infty} \varepsilon_{n} i^{-n} J_{n}(K r) \cos n \theta
$$

where $\varepsilon_{0}=1, \varepsilon_{n}=2(n=1,2, \ldots)$, and $J_{n}$ is a Bessel function. The perturbation pressure may be expressed as a functional of $\chi$ through the application of Green's second theorem to $\phi$ and the Green's function determined by

$$
\left(\nabla^{2}+\varepsilon^{2}\right) G=-\delta(\mathbf{r}-\rho)
$$


where $\delta$ is Dirac's delta function, and $\mathbf{r}$ and $\rho$ are dimensionless vectors; $c f$. Levine and Schwinger [1]. The end result may be expressed in the form

$$
\phi=\sum_{n=0}^{\infty} \varepsilon_{n} i^{-n} \phi_{n}(r, z) \cos n \theta,
$$

where

$$
\begin{gathered}
\phi_{n}=J_{n}(K r) e^{-K z}-\left.\int_{0}^{\infty} \partial_{\rho} G_{n}(r, z ; \rho, \zeta)\right|_{\rho=1} \chi_{n}(\zeta) d \zeta, \\
G_{n}(r, z ; \rho, \zeta)=\frac{1}{2 \pi} \int_{-\infty}^{\infty}\left\{\begin{array}{l}
I_{n}(\kappa \rho) K_{n}(\kappa r) \\
I_{n}(\kappa r) K_{n}(\kappa \rho)
\end{array}\right\} e^{i k(\zeta-z)} d k \quad(r \gtrless \rho), \\
\kappa=\left(k^{2}-\varepsilon^{2}\right)^{1 / 2}, \\
\chi_{n}(z)=\frac{i^{n}}{2 \pi} \int_{0}^{2 \pi} \chi(z, \theta) \cos n \theta d \theta,
\end{gathered}
$$

and $I_{n}$ and $K_{n}$ are modified Bessel functions. The parameter $\kappa$ is an analytic function of the complex variable $k$ in a complex plane (see Figure 2) cut from $\pm \varepsilon$ to $\pm \alpha \mp i \infty$ (alternative signs are vertically ordered) and has the limiting $(\varepsilon \rightarrow 0)$ value $|k|$ on the real axis. The branch points tend to the real axis in the limit $\beta \downarrow 0$, in which limit $\arg \kappa=\frac{1}{2} \pi$ for any of $-\alpha<k<\alpha, \arg k=+\frac{1}{2} \pi$, or $\arg k=-\frac{1}{2} \pi$.

That (2.3) satisfies (1.7) follows through separation of variables; that it satisfies (1.3) is evident from the exponential decay of $K_{n}(\kappa r)$ as $\kappa r \rightarrow \infty$; that it satisfies (1.5) and (1.6a) follows from Fourier's theorem and the Wronskian relation

$$
I_{n}^{\prime}(\kappa) K_{n}(\kappa)-I_{n}(\kappa) K_{n}^{\prime}(\kappa)=\kappa^{-1} \text {. }
$$

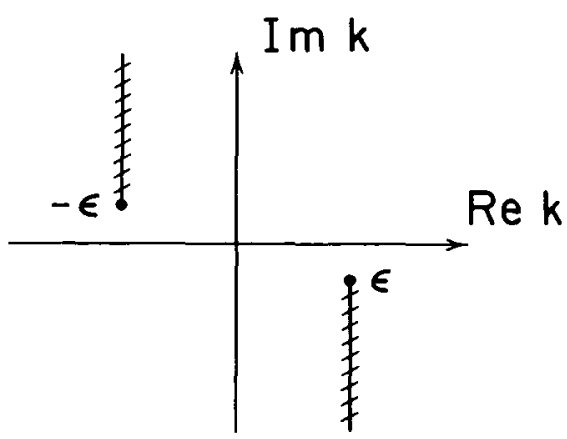

Figure 2. Complex- $k$ plane with cuts for $\kappa=\left(k^{2}-\varepsilon^{2}\right)^{1 / 2}, \varepsilon=\alpha-i \beta$. 
It remains to satisfy the kinematic boundary condition (1.4). Combining (2.3) and (2.4) therein, we obtain the Wiener-Hopf integral equation

$$
\begin{aligned}
& \int_{0}^{\infty} g_{n}(z-\zeta) \chi_{n}(\zeta) d \zeta=-K J_{n}^{\prime}(K) e^{-K z} \equiv \psi_{n}(z) \\
&(n=0,1,2, \ldots, 0<z<\infty)
\end{aligned}
$$

with the kernel

$$
\begin{aligned}
g_{n}(z-\zeta) & =-\left.\partial_{r} \partial_{\rho} G_{n}(r, z ; \rho, \zeta)\right|_{r=\rho=1} \\
& =-\frac{1}{2 \pi} \int_{-\infty}^{\infty} \kappa^{2} I_{n}^{\prime}(\kappa) K_{n}^{\prime}(\kappa) e^{i k(\zeta-z)} d k .
\end{aligned}
$$

\section{Wiener-Hopf solution}

Following Noble [5], we introduce the sectional transforms

$$
F_{+}(k)=(2 \pi)^{-1 / 2} \int_{0}^{\infty} f(z) e^{i k z} d z, \quad F_{-}(k)=(2 \pi)^{-1 / 2} \int_{-\infty}^{0} f(z) e^{i k z} d z
$$

and extend (2.9) into $-\infty<z<0$. Fourier-transforming the extended equation with the aid of the convolution theorem, we obtain

$$
-\kappa^{2} I_{n}^{\prime}(\kappa) K_{n}^{\prime}(\kappa) \mathrm{X}_{n+}(k)=\Psi_{n+}(k)+\Psi_{n-}(k),
$$

where $\mathrm{X}_{n \pm}$ is the sectional transform of $\chi_{n}(z)$ in $z \gtrless 0$ and similarly for $\Psi_{n \pm}$. It follows from $(1.6 \mathrm{a}, \mathrm{b})$ that

$$
\mathrm{X}_{n-}(k) \equiv 0, \quad \mathrm{X}_{n+}(k)=O\left(k^{-3 / 2}\right) \quad(k \rightarrow \infty),
$$

and from (2.9) that

$$
\Psi_{n+}(k)=-(2 \pi)^{-1 / 2} K J_{n}^{\prime}(K)(K-i k)^{-1} .
$$

The functions $\mathrm{X}_{n+}(k)$ and $\Psi_{n-}(k)$ are unknown, although only $\mathrm{X}_{n+}(k)$ is required in the present context.

We consider first the axisymmetric problem, $n=0$. Dropping the subscript $n=0$, factoring $\kappa^{2}=(k-\varepsilon)(k+\varepsilon)$, introducing and factoring

$$
H(k) \equiv-2 I_{0}^{\prime}(\kappa) K_{0}^{\prime}(\kappa)=2 I_{1}(\kappa) K_{1}(\kappa)=H_{+}(k) H_{-}(k),
$$

where $H_{ \pm}(k)$ (see below) is analytic in $\operatorname{Im} k \gtrless \mp \beta$, and dividing (3.2) through by $\frac{1}{2}(k+\varepsilon) H_{-}(k)$, we rewrite (3.2) in the form

$$
(k-\varepsilon) H_{+}(k) X_{+}(k)=2\left\{(k+\varepsilon) H_{-}(k)\right\}^{-1}\left\{\Psi_{+}(k)+\Psi_{-}(k)\right\} .
$$


Separating out the contribution of the pole at $k=-i K$ to the right-hand side of (3.6), we obtain

$$
\begin{gathered}
(k-\varepsilon) H_{+}(k) \mathrm{X}_{+}(k)-2\left\{(-i K+\varepsilon) H_{-}(-i K)\right\}^{-1} \Psi_{+}(k) \\
=2\left[\left\{(k+\varepsilon) H_{-}(k)\right\}^{-1}-\left\{(-i K+\varepsilon) H_{-}(-i K)\right\}^{-1}\right] \Psi_{+}(k) \\
+2\left\{(k+\varepsilon) H_{-}(k)\right\}^{-1} \Psi_{-}(k) \equiv F(k) \quad(0<\operatorname{Im} k<\beta) .
\end{gathered}
$$

The left-hand side of (3.7) is analytic in the half-plane $\operatorname{Im} k>0$ (i.e. above the poles of $\mathrm{X}_{+}$at $k=0$ and $\Psi_{+}$at $k=-i K$ and the branch point of $H_{+}$at $k=\varepsilon$ ); the right-hand side is analytic in the half-plane $\operatorname{Im} k<\beta$ (i.e. below the branch point of $H_{-}$at $k=\varepsilon$ ). Moreover, it can be shown (cf. Levine and Schwinger [1, Appendix B]) that

$$
H_{+}(k) \sim(-i k)^{-1 / 2} \quad(k \rightarrow \infty, \operatorname{Im} k>0),
$$

which, in conjunction with (3.3b) and (3.4), implies that the left-hand side of (3.7) is $O\left(k^{-1}\right)$ as $k \rightarrow \infty$ in the upper half-plane. It then follows from Liouville's theorem that the left- and right-hand sides of (3.7) are analytic representations, with the overlapping domain $0<\operatorname{Im} k<\beta$, of the integral function $F(k)=0$. Solving for $\mathrm{X}_{+}$and letting $\varepsilon \rightarrow 0$, we obtain

$$
\begin{array}{r}
\mathrm{X}_{+}(k)=i(2 / \pi)^{1 / 2} J_{1}(K)\left\{H_{-}(-i K) H_{+}(k) k(K-i k)\right\}^{-1} \\
(\varepsilon=0, \operatorname{Im} k>0) .
\end{array}
$$

Setting $n=0$ and $J_{0}^{\prime}=-J_{1}$ in (3.4), substituting the result into (3.9), and inverting the resulting Fourier transform (recall that $X_{-} \equiv 0$ ), we obtain

$$
\begin{aligned}
\chi(z) & =(2 \pi)^{-1 / 2} \int_{-\infty+i c}^{\infty+i c} \mathrm{X}_{+}(k) e^{-i k z} d k \quad(c>0) \\
& =\frac{i J_{1}(K)}{\pi H_{-}(-i K)} \int_{-\infty+i c}^{\infty+i c} \frac{e^{-i k z} d k}{k(K-i k) H_{+}(k)} .
\end{aligned}
$$

The factoring of $H(k)$ follows Noble [5, Section 3.4], in whose notation $H(k)=K(\alpha)$, but the branch cuts for the present $\kappa$ differ from those for his $\gamma$ in consequence of the respective choices $\exp (i \omega t)$ and $\exp (-i \omega t)$ for the complex time dependence. The initial factoring (which follows from Cauchy's integral theorem) yields

$$
\begin{aligned}
\log H_{ \pm}(k) & = \pm \frac{1}{2 \pi i} \int_{-\infty}^{\infty} \frac{\log H(t) d t}{t-k} \quad(\operatorname{Im} k \gtrless 0) \\
& =\mp \frac{i k}{\pi} \int_{0}^{\infty} \frac{\log H(t) d t}{t^{2}-k^{2}},
\end{aligned}
$$

in which the principal branch of the logarithm is implicit. The improper integral in (3.1 la) is to be interpreted as the limit of the integral from $t=-T$ to $t=T$ as 
$T \rightarrow \infty$, and (3.11b) follows from (3.11a) by virtue of $H(-t)=H(t)$. Letting $k$ tend to the real axis and indenting the contour for $H_{ \pm}$below/above $t=k$, we obtain

$$
H_{ \pm}(k)=H^{1 / 2}(k) \exp \left\{\mp \frac{i k}{\pi} f_{0}^{\infty} \frac{\log H(t) d t}{t^{2}-k^{2}}\right\} \quad(\operatorname{Im} k=0),
$$

in which the integral is a Cauchy principal value.

We turn now to the evaluation of $P$, as given by (1.9). Letting $f=\chi$ and $\operatorname{Im} k>0$ in (3.1a), integrating by parts, and letting $k \rightarrow 0$, we obtain

$$
P=-(2 \pi)^{1 / 2} \lim _{k \rightarrow 0} i k \mathrm{X}_{+}(k) \quad(\operatorname{Im} k=0+) \text {. }
$$

Substituting (3.9) into (3.13) and substituting $H_{+}(0)=1$ and $H_{-}(-i K)$ from (3.12) and (3.11b), respectively, we obtain

$$
\begin{aligned}
P & =2 J_{1}(K)\left\{K H_{+}(0) H_{-}(-i K)\right\}^{-1} \\
& =\frac{2 J_{1}(K)}{K} \exp \left\{-\frac{K}{\pi} \int_{0}^{\infty} \frac{\log H(t)}{t^{2}+K^{2}} d t\right\},
\end{aligned}
$$

which is plotted in Figure 3. The zeros of $J_{1}(K)$ correspond to transverse resonances of the duct. The present result for $P$ is close to Simon's [6, Figure 7] ( $P \equiv K_{A}$ and $\mu \equiv K$ in Simon's notation) for $h / a=4$ but falls below that plot for $K>1.3$.

Letting $K \rightarrow 0$ and $K \rightarrow \infty$, we obtain

$$
P=1+C K+O\left(K^{2}\right) \quad(K \rightarrow 0), \quad C=-\frac{1}{\pi} \int_{0}^{\infty} \frac{\log H(t)}{t^{2}} d t=0.6133 \ldots
$$

and

$$
P \sim(8 / \pi)^{1 / 2} K^{-1} \sin \left(K-\frac{1}{4} \pi\right) \quad(K \rightarrow \infty) .
$$

The solution of (3.2) for $n=1,2, \ldots$ follows (3.5)-(3.9) with only minor changes. In place of (3.5), we choose

$$
H_{n}(k) \equiv-2 n^{-1} \kappa^{2} I_{n}^{\prime}(\kappa) K_{n}^{\prime}(\kappa)=H_{n+}(k) H_{n-}(k) \quad(n>0),
$$

which is normalized to $H_{n}=1$ at $\kappa=0$. The counterpart of (3.6) then is obtained by deleting the factors $k \pm \varepsilon$ and yields

$$
\mathrm{X}_{n+}(k)=2\left\{n H_{n-}(-i K) H_{n+}(k)\right\}^{-1} \Psi_{n+}(k) \quad(\varepsilon=0, \operatorname{Im} k \geqslant 0),
$$

where the factors $H_{n \pm}$ are given by (3.11) and (3.12) with $H$ replaced by $H_{n}$ therein, and $\Psi_{n+}$ is given by (3.4). It follows from (3.18) that $X_{n+}(0)$ is bounded, and hence that $\chi_{n}(\infty)=0$, as anticipated in (1.9). 


\section{Approximate solution}

The interpolation

$$
H(k) \div\left(1+k^{2}\right)^{-1 / 2} \quad(\varepsilon=0)
$$

between the limits $H(0)=1$ and $H(k) \sim 1 / k$ is in error by less than $5 \%$ in $0<k<\infty$. The corresponding approximations

$$
H_{ \pm}(k) \doteqdot(1 \mp i k)^{-1 / 2} \quad(\operatorname{lm} k \gtrless \mp 1)
$$

may be obtained either by factoring (4.1) directly and then invoking analytic continuation or by substituting (4.1) into (3.11b).

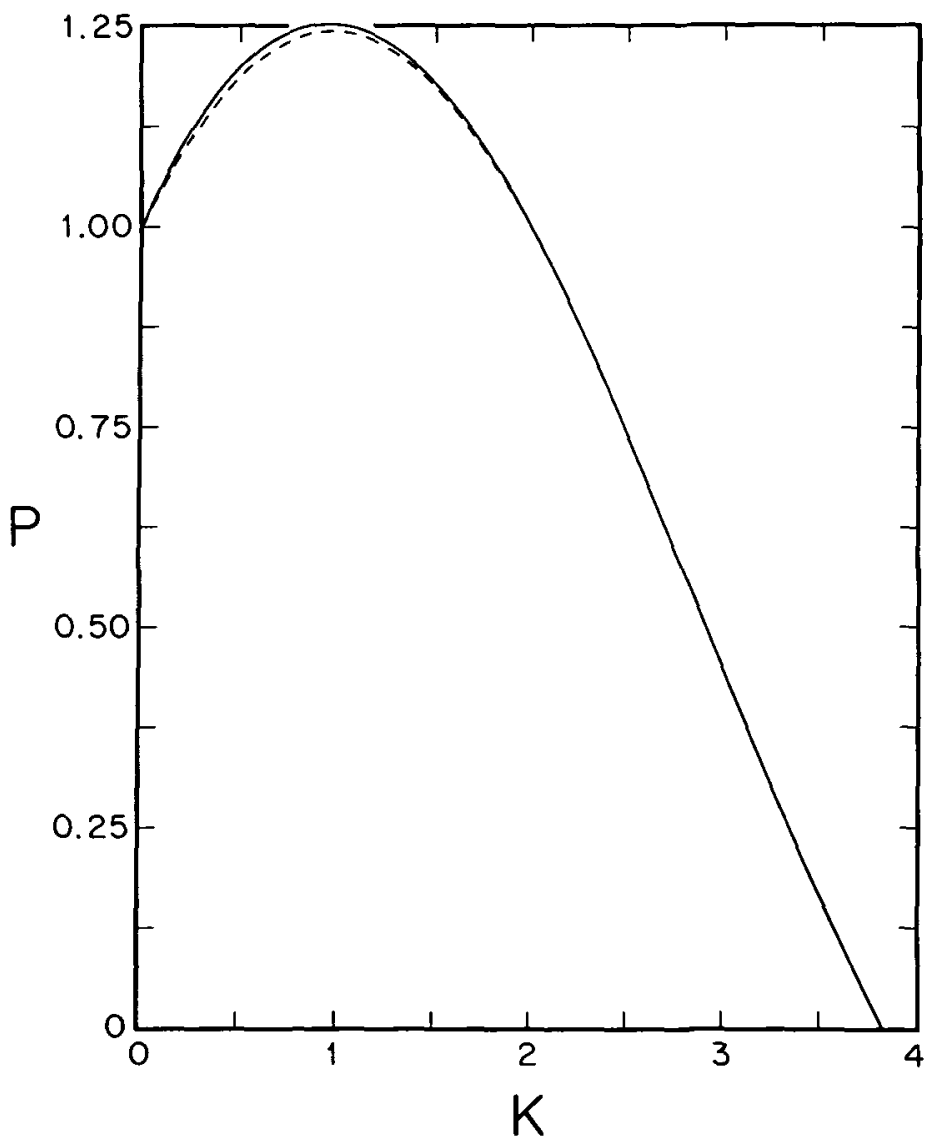

Figure 3. The pressure amplification factor $P$, as given by (3.14) (-) and (4.3) (--). 
Substituting (4.2) into (3.14a), we obtain

$$
P \doteqdot 2 K^{-1}(1+K)^{1 / 2} J_{1}(K)
$$

which is compared with (3.14b) in Figure 3. It follows from this comparison that the maximum error associated with (4.3) is $1.2 \%$.

Substituting (4.2) into (3.10b) and invoking (4.3), we obtain

$$
\begin{aligned}
\chi(z) & \doteqdot \frac{i K P}{2 \pi} \int_{-\infty+i c}^{\infty+c} \frac{(1-i k)^{1 / 2} e^{-\imath k z} d k}{k(K-i k)} \\
& =P\left[\operatorname{erf}\left(z^{1 / 2}\right)-(1-K)^{1 / 2} e^{-K z} \operatorname{erf}\left\{(1-K)^{1 / 2} z^{1 / 2}\right\}\right] \quad(z \geqslant 0)
\end{aligned}
$$

where erf signifies the error function [the integral in (4.4a) may be evaluated with the aid of a table of inverse Laplace transforms after letting $s=-i k$ or directly by contour integration]. Letting $z \downarrow 0$ and $z \uparrow \infty$ in (4.4b), we obtain

$$
\chi(z) \doteqdot 2 K P(z / \pi)^{1 / 2} \quad(z \downarrow 0)
$$

and

$$
\chi(z) \sim p\left\{\begin{array}{c}
1-(1-K)^{1 / 2} e^{-K z} \\
1-(\pi z)^{-1 / 2} e^{-z} \\
1+\frac{1}{2} K(K-1)^{-1}\left(\pi z^{3}\right)^{-1 / 2} e^{-z}
\end{array}\right\} \quad(K \leqq 1, z \rightarrow \infty)
$$

The counterparts of (4.1) and (4.2) for $n>0$ are, from (3.17),

$$
H_{n}(k) \doteqdot\left\{1+(k / n)^{2}\right\}^{1 / 2} \quad(\varepsilon=0)
$$

and

$$
H_{n \pm}(k)=\{1 \mp(i k / n)\}^{1 / 2} \text {. }
$$

Substituting (4.8) into (3.18) and proceeding as above, we obtain

$$
\chi_{n}(z) \div-2 K\left(n^{2}-K^{2}\right)^{-1 / 2} J_{n}^{\prime}(K) e^{-K z} \operatorname{erf}\left\{(n-K)^{1 / 2} z^{1 / 2}\right\}
$$

\section{Acknowledgement}

This work was supported in part by the Physical Oceanography Division, National Science Foundation, NSF Grant OCE77-24005, and by a contract with the Office of Naval Research. 


\section{References}

[1] H. Levine and J. Schwinger, "On the radiation of sound from an unflanged circular pipe", Phys. Rev. 73 (1949), 383-406.

[2] M. J. Lighthill, Waves in fluids (Cambridge University Press, 1978).

[3] M. J. Lighthill, "Two-dimensional analyses related to wave-energy extraction by submerged resonant ducts", J. Fluid Mech. 91 (1979), 253-317.

[4] J. W. Miles, "Surface wave diffraction by a periodic row of submerged ducts", J. Fluld Mech. 128 (1983), $155-180$.

[5] B. Noble, Methods based on the Wiener-Hopf technique for the solution of partial differential equations (Pergamon Press, 1958).

[6] M. J. Simon, "Wave-energy extraction by a submerged cylindrical resonant duct", J. Fluid Mech. 104 (1981), 159-187. 